\title{
Estimating restricted mean survival time and expected life-years lost in the presence of competing risks within flexible parametric survival models
}

\section{CURRENT STATUS: UNDER REVIEW}

BMC Medical Research Methodology $\triangle$ BMC series

Sarwar Islam Mozumder

University of Leicester

sarwar.islam@gmail.comCorresponding Author

ORCiD: https://orcid.org/0000-0001-9644-7525

Paul Lambert

University of Leicester College of Medicine Biological Sciences and Psychology

Mark Rutherford

University of Leicester College of Medicine Biological Sciences and Psychology

\section{DOI:}

$10.21203 / \mathrm{rs} .2 .23839 / \mathrm{v} 1$

\section{SUBJECT AREAS}

Health Economics \& Outcomes Research

\section{KEYWORDS}

competing risks, restricted mean survival time, restricted mean life 1 time, flexible parametric model, life-years lost, survival analysis 
Abstract

We present various measures, specifically the expected life-years list due to a cause of death, that can be predicted for a specific covariate pattern. These can also be summarised at the populationlevel using standardisation to obtain marginal measures. The restricted mean survival time (RMST) measure can be obtained in the presence of competing risks using Royston-Parmar flexible parametric survival models (FPMs). Royston-Parmar FPMs can be fitted on either the cause-specific hazards or cumulative incidence scale in the presence of competing risks. An advantage of modelling within this framework for competing risks data is the ease at which other alternative predictions to the (cause-specific or subdistribution) hazard ratio can be obtained. The RMST estimate is one such measure. This has an attractive interpretation, especially when the proportionality assumption is violated. In addition to this, compared to similar measures, fewer assumptions are required and it does not require extrapolation. Furthermore, one can easily obtain the expected number of life-years lost, or gained, due to a particular cause of death, which is a further useful prognostic measure. We describe estimation of RMST after fitting a FPM on either the log-cumulative subdistribution, or causespecific hazards scale. As an illustration of reporting such measures to facilitate interpretation of a competing risks analysis, models are fitted to English colorectal data.

\section{Full-text}

Due to technical limitations, full-text HTML conversion of this manuscript could not be completed. However, the manuscript can be downloaded and accessed as a PDF.

\section{Figures}




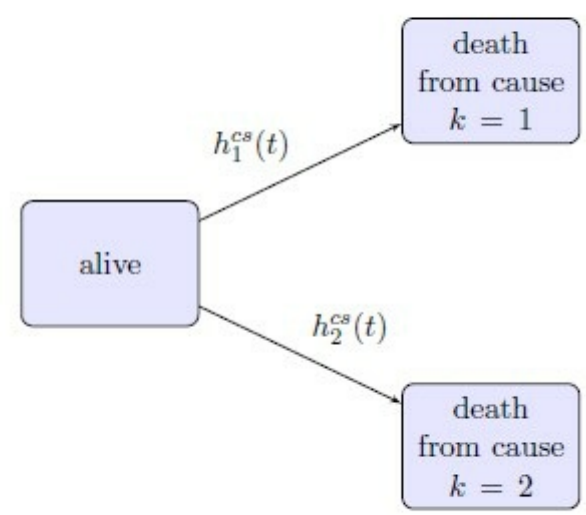

(a) Transitions described by the cause-specific hazard rate. The typical competing risks scenario.
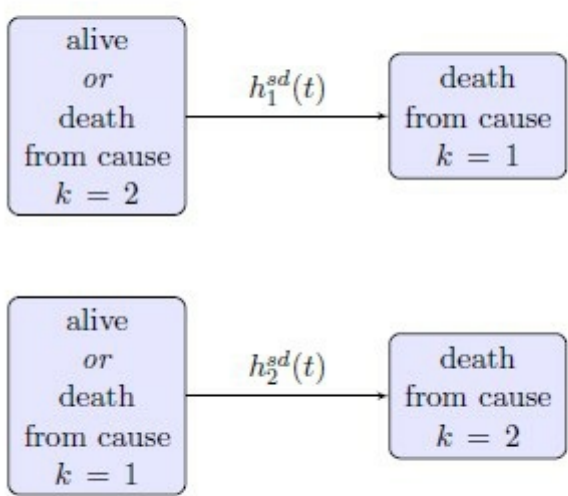

(b) Transitions described by the subdistribution hazard rate.

Figure 1

(a) Transitions described by the cause- specic hazard rate. The typical competing risks scenario. (b) Transitions described by the subdistribu- tion hazard rate. Schematics of transitions from an initial state to one of $\mathrm{K}=2$ absorbing states that correspond to a particular cause of death in the presence of competing risks. 

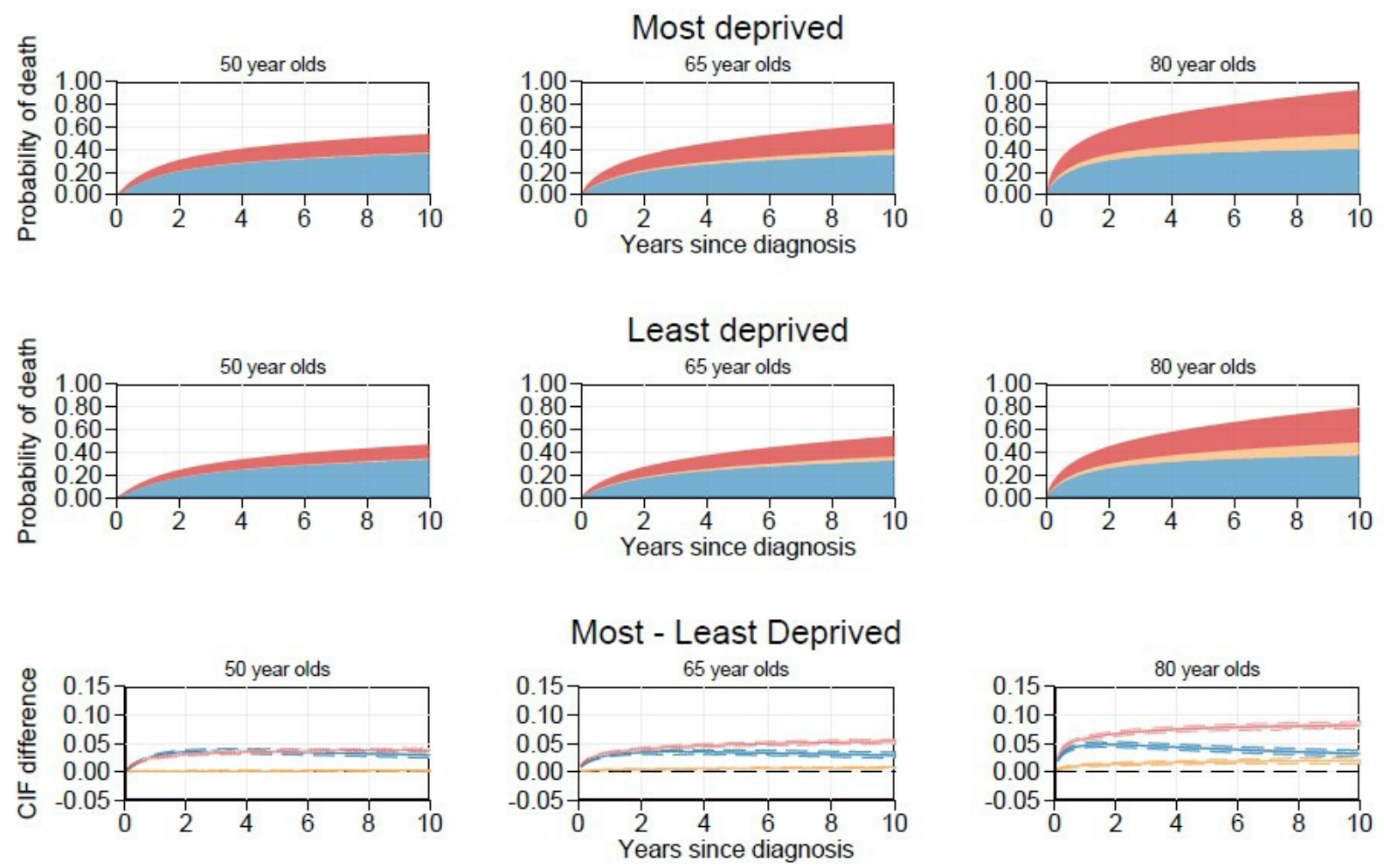

\section{$\begin{array}{lll}\text { Cancer } & \text { CVD } & \text { Other causes }\end{array}$}

Figure 2

Stacked cause-specic cumulative incidence functions (CIFs) for the most (top row) and least (middle row) deprived groups for male patients at 50, 65 and 80 years old at diagnosis. CIF dierences (most - least deprived) are presented on the bottom row with associated 95\% condence intervals (region enclosed by dashed lines). 

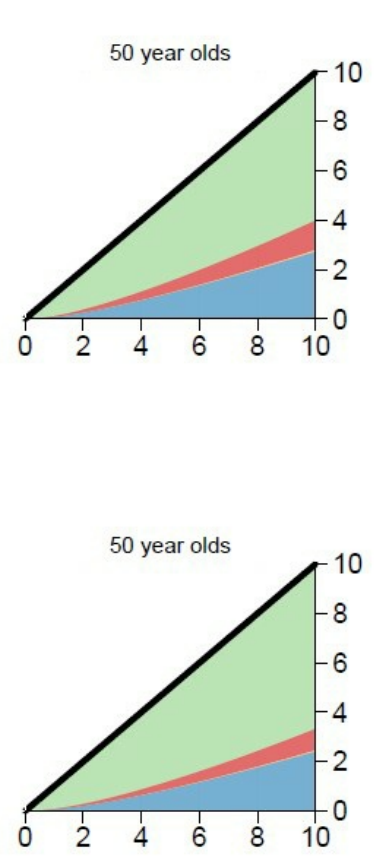

Most deprived

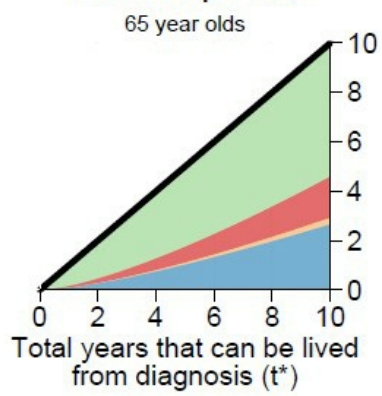

\section{Least deprived}

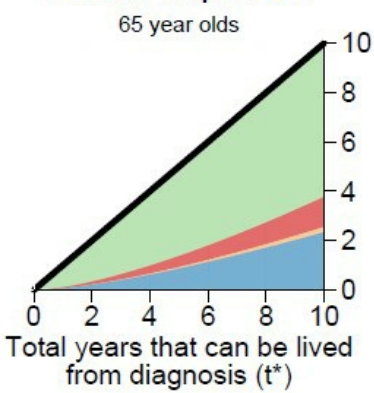

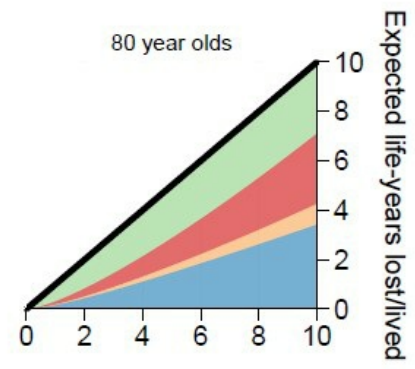

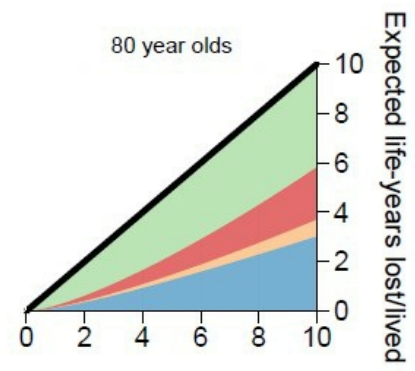

Figure 3

Stacked plots of expected life-years lost partitioned by each cause of death and total lifeyears lived for the most deprived (top row) and least deprived (bottom row) for male patients aged 50, 65 and 80 years old at diagnosis. These are presented at different years from diagnosis, $\mathrm{t}^{*}$. 
Cause-specific ClFs standardised by age and sex
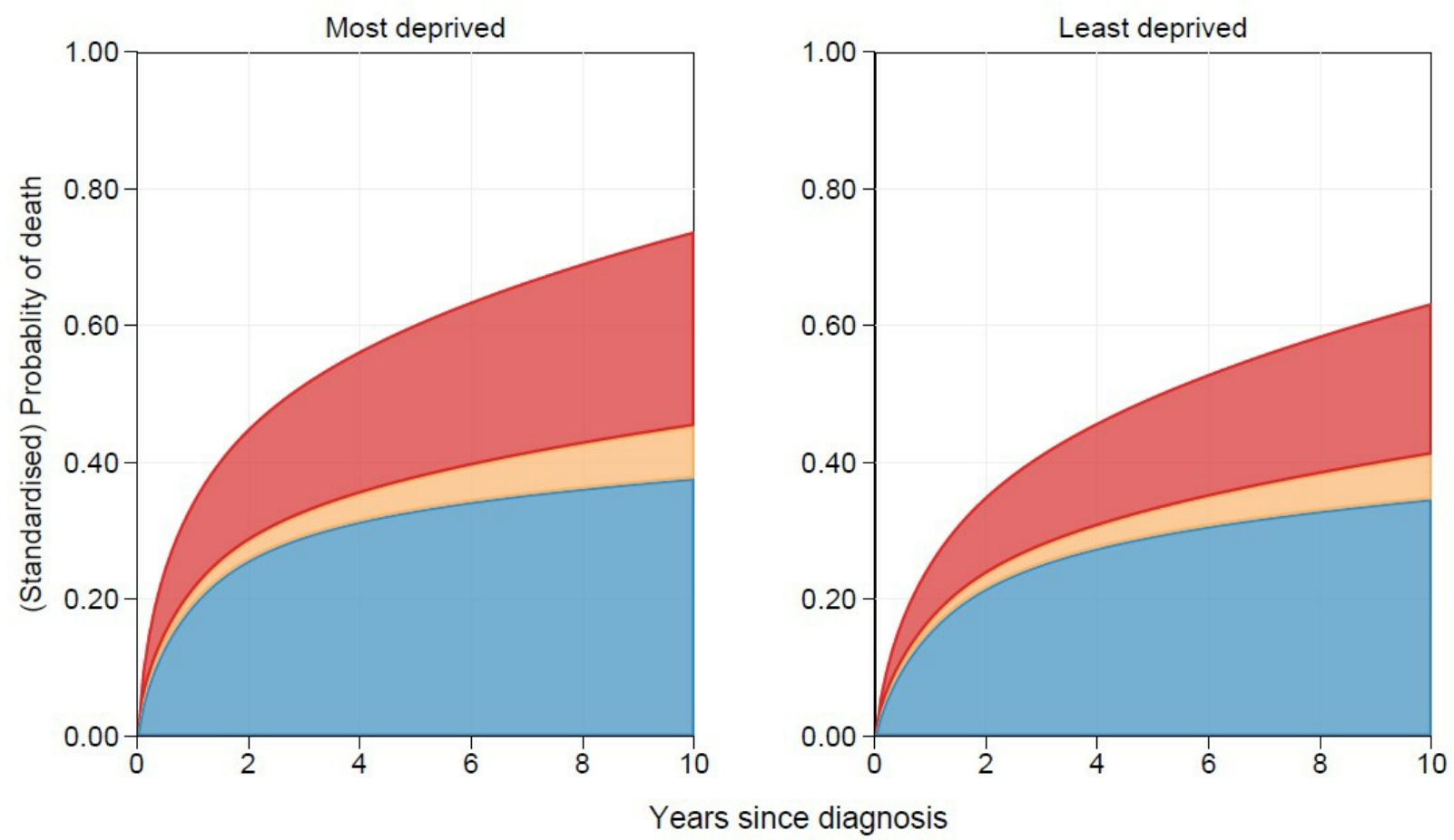

\section{\begin{tabular}{|l|l|l}
\hline CVD & Other causes $\square$ Cancer
\end{tabular}}

Figure 4

Estimated cause-specic cumulative incidence functions (CIFs) stacked for each cause of death. Predictions are standardised by age and sex and obtained for the most (left) and least (right) deprived. 

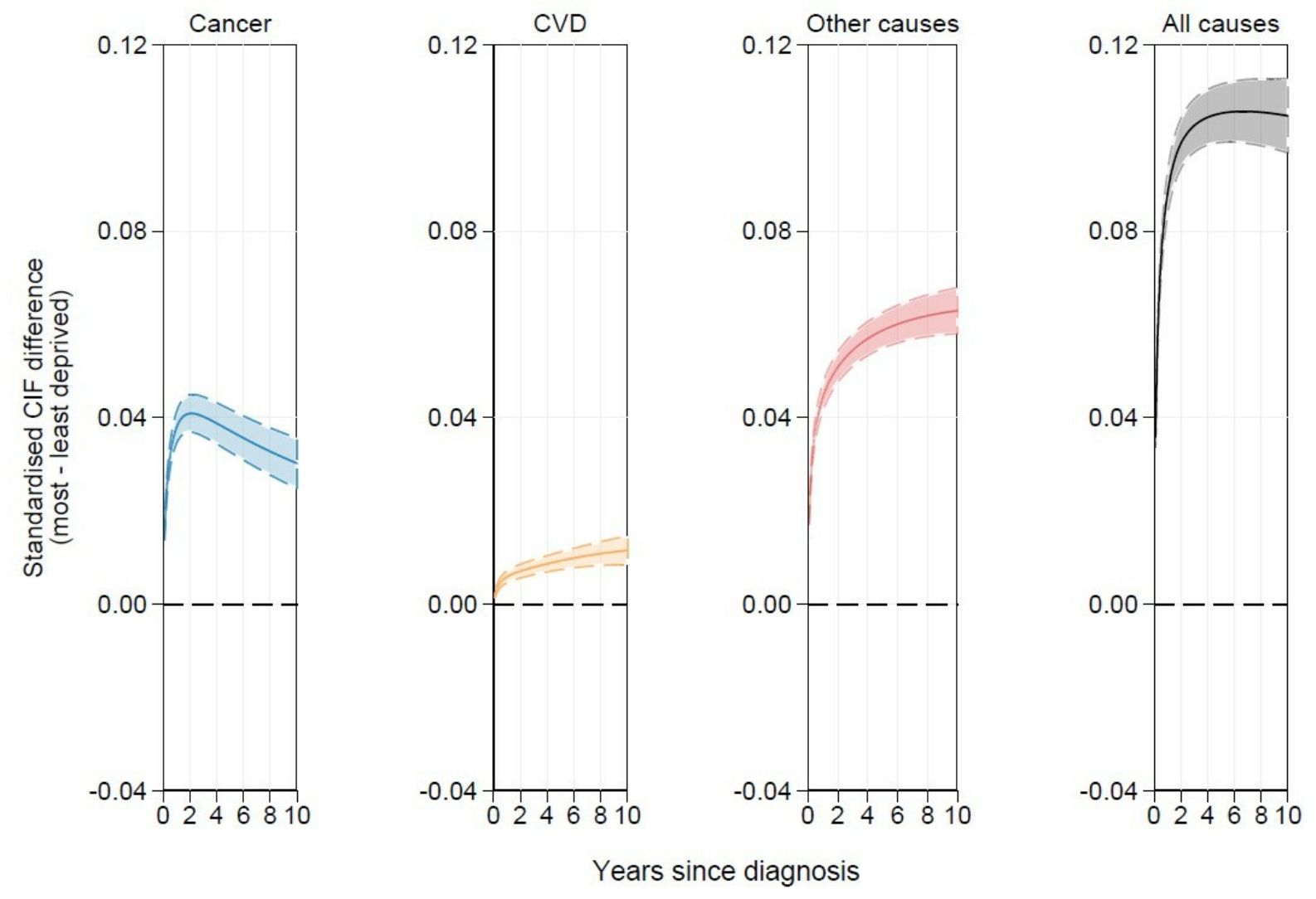

Figure 5

Predicted differences in cause-specic cumulative inci- dence functions (CIF) between the most and least deprived patients with associated 95\% condence intervals (dashed lines).

Estimates are standardised by age and sex. 

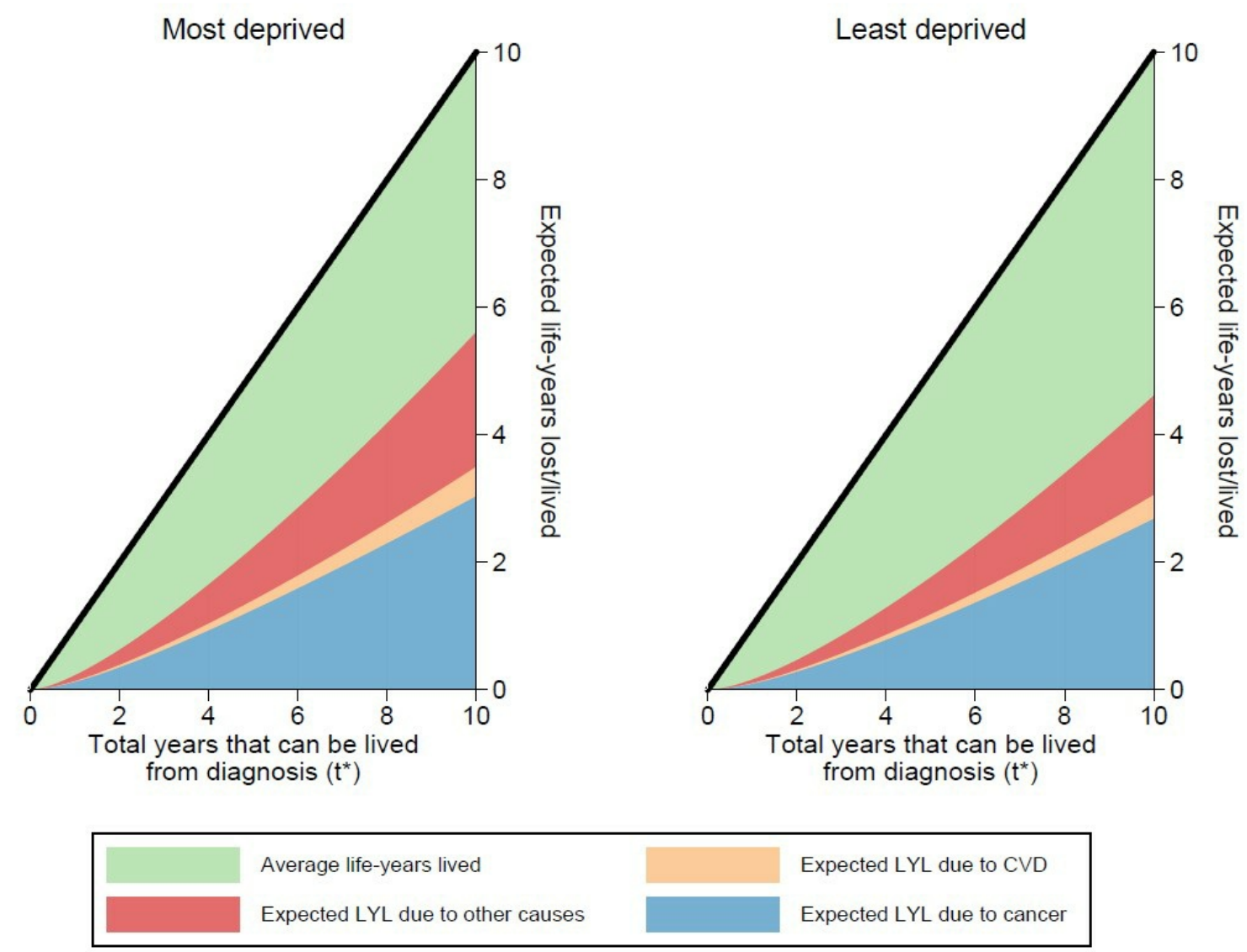

Figure 6

Stacked plots of expected life-years lost partitioned by each cause of death and total lifeyears lived for the most deprived (left) and least deprived (right) patients standardised by age and sex. These are presented for different years from diagnosis, $t^{*}$.

\section{Supplementary Files}

This is a list of supplementary files associated with this preprint. Click to download.

refs_02Feb19.bib

rmft_age.log.tex

cov_stand2.log.tex

model.log.tex

cov_age.log.tex

cif_stand2.log.tex

cif_age.log.tex

code_appendix.tex

rmft_stand2.log.tex

bmc-mathphys.bst

stata.sty

model2.log.tex 
stset.log.tex

stcrprep.log.tex

bmcart.cls 